\title{
Brillouin echo-distributed sensing based on differential phase-shift keying technique
}

\author{
Min Won Lee ${ }^{1}$, Birgit Stiller ${ }^{1}$, Jérôme Hauden ${ }^{2}$, \\ Hervé Maillotte $^{1}$, Luc Thévenaz ${ }^{3}$, and Thibaut Sylvestre ${ }^{1}$ \\ ${ }^{1}$ Institut FEMTO-ST, Université de Franche-Comté, CNRS UMR 6174, F-25030 Besançon, France \\ 2 PHOTLINE Technologies, F-25001 Besançon, France \\ ${ }^{3}$ Institute of Electrical Engineering, Ecole Polytechnique Fédérale de Lausanne, CH-1015, Lausanne, Switzerland \\ thibaut.sylvestre@univ-fcomte.fr
}

\begin{abstract}
In this letter, we demonstrate experimentally Brillouin echo-distributed sensing based on differential phase-shift keying technique. A positive and negative pulse is applied to a single Mach-Zehnder modulator to achieve a resolution of $5 \mathrm{~cm}$.
\end{abstract}

OCIS codes: $290.5900,060.2370$

\section{Introduction}

Over the last two decades, Brillouin scattering has widely been studied for distributed sensing application using optical fibres [1-3]. Compared to other techniques, Brillouin Optical Time-Domain Analysis (BOTDA) has attracted particular attention for distributed sensing of temperature and/or strain in petrol and civil engineering industries. This technique offers fast measurement and long-haul distributed sensing by measuring Brillouin frequency shift (BFS) produced by temperature and/or strain in optical fibres [4]. Basically, the technique uses a pump-probe counter-propagative configuration with a pulse longer than $10 \mathrm{~ns}$. However, its spatial resolution is limited to $1 \mathrm{~m}$ due to the acoustic decay time of $10 \mathrm{~ns}$. In order to overcome this limit, other techniques have been developed [5, 6]. One of the techniques is called Brillouin Echo Distributed Sensing (BEDS) [7]. This technique uses a short $\pi$-phase pulse in addition to a long intensity pulse and the centimetre resolution is achieved by the phase pulse duration. Despite of the advantage over spatial resolution, the conventional BEDS systems exploit two modulators: a phase modulator for generating $\pi$-phase pulses and an intensity modulator for generating long intensity pulses. Moreover, as the phase pulse must be positioned at the end of the intensity pulse, it needs a sophisticate adjustment of the delay between the pulses.

In this work, we have developed a new technique for BEDS systems in which a $\pi$-phase pulses is directly generated using a single intensity modulator by Differential Phase-Shift Keying (DPSK) technique. A long positive pulse is followed by a short negative pulse and this positive-negative shape pulse is applied to the modulator to generate a phase shift at the end of the long intensity pulse. With this technique, centimetre spatial resolution is achieved in our BEDS system. In order to demonstrate it, two different fibres are spliced with a $5 \mathrm{~cm}$-protection sleeve. The results will be compared with the results obtained in conventional BEDS system.

\section{Experimental setup}

DPSK technique is widely used in telecom industry to transmit bit-sequence messages in form of $\pi$-phase shift using an intensity modulator, i.e. Mach-Zehnder modulator (MZM). When MZM is driven by a voltage between 0 and a half-wave voltage $\left(V_{\pi}\right)$, the output signal phase of the modulator is not modified. On the other hand, the phase of the output signal is shifted by $\pi$ for a voltage between $V_{\pi}$ and $2 V_{\pi}$ [8]. Therefore, when a negative pulse of $V_{\pi}$ is applied to the modulator just after a positive pulse at the minimum bias point as illustrated in Fig. 1(a), the output optical intensity remains unchanged whilst the phase is shifted by $\pi$ at the negative pulse part, or vice-versa. Because of the difficulty of such a pulse generation in the work, the positive long and negative short pulses are applied to the DC and RF inputs of the modulator, respectively. The DC input is adapted to $50 \Omega$ and the DC is set at the minimum bias point of the MZM. As the pulses are in form of intensity, the delay between the pulses is readily adjusted at the output of the MZM. Figure 1(b) exhibits the modulator output of the two intensity pulses. A 30-ns positive pulse with a zero-phase is followed by a 500-ps negative pulse with a $\pi$-shifted phase pulse. The intensity is almost constant except a small transition from the positive pulse to the negative pulse. The 500-ps duration of the short pulse defines a spatial resolution of $5 \mathrm{~cm}$ in our BEDS system. 

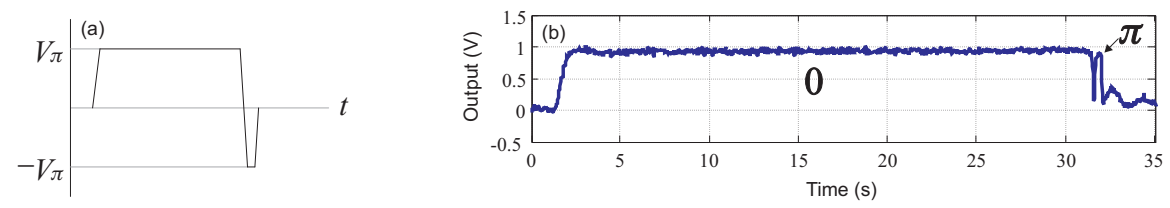

Fig. 1. The modulator output of two intensity pulses. The durations of the long and short pulses are $30 \mathrm{~ns}$ and $500 \mathrm{ps}$, respectively. The phase is shifted by $\pi$ for $500 \mathrm{ps}$ at the end.

This dual-phase state intensity pulse is used as the pump in the experimental setup shown in Fig. 2 schematically. The setup is based on BOTDA system with an additional negative pulse in the pump. The CW emission of a distributed feedback (DFB) laser at $1550 \mathrm{~nm}$ is splitted into the pump and probe arms. The DC bias of an MZM (Photline MXPE series) is adjusted to be at the minimum bias point. The pulse is amplified by an Erbium-doped fibre amplifier (EDFA) with a peak power of $1.3 \mathrm{~W}$, polarisation-scrambled to average out polarisation-sensitive Brillouin gain and injected into a fibre under test via a circulator. The Brillouin frequency-shifted (BFS) probe is generated by the second MZM (Photline MXPE series) with a carrier suppression of $38 \mathrm{~dB}$ by adjusting the DC bias. The probe power is set to $1 \mathrm{~mW}$ by another EDFA. The probe is then injected into the fibre after an isolator. A fibre Bragg grating (FBG) is used to select the gain obtained by the pump at the BFS. The gain is retrieved by a photodetector and then recorded as time traces by a digital oscilloscope. By sweeping the frequency of the probe, the gain of the fibre is mapped as a function of frequency.

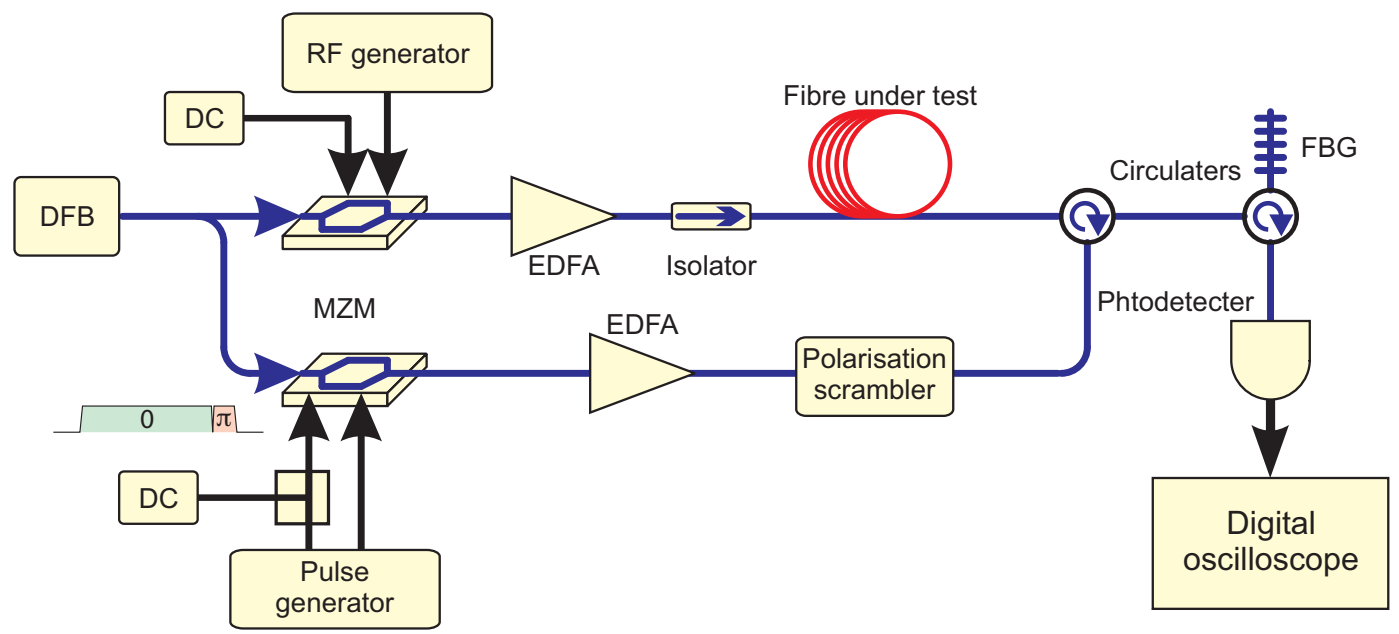

Fig. 2. Experimental setup of our simplified BEDS system using a single MZM for the pump. EDFA: Erbium-doped fibre amplifier, FBG: Fibre Bragg grating.

\section{Experimental results}

In order to demonstrate the performance of the technique developed in the work, a 1-m single mode fibre (SMF) is spliced with a 1-m fibre with high numerical aperture (HNA). The splice point is protected by a heat-shrinking sleeve of $5 \mathrm{~cm}$. For comparison, distributed measurements of the splice segment have been done in both BEDS systems. Figure 3(a) shows the distributed measurement obtained in the conventional BEDS system using a $\pi$-phase pulse with a pulse width of $500 \mathrm{ps}$. It clearly shows the splice segment of $5 \mathrm{~cm}$ between the fibres at a frequency shift of 10.55 GHz. The BFS of the HNA and SMF are measured as $10.67 \mathrm{GHz}$ and $10.85 \mathrm{GHz}$, respectively. Figure 3(b) displays the mapping using the technique developed in the work. It also shows clearly the 5 -cm splice segment and the 10.55-GHz frequency shift. Therefore, it is evident that our technique manifestes good performances as in standard BEDS systems. 

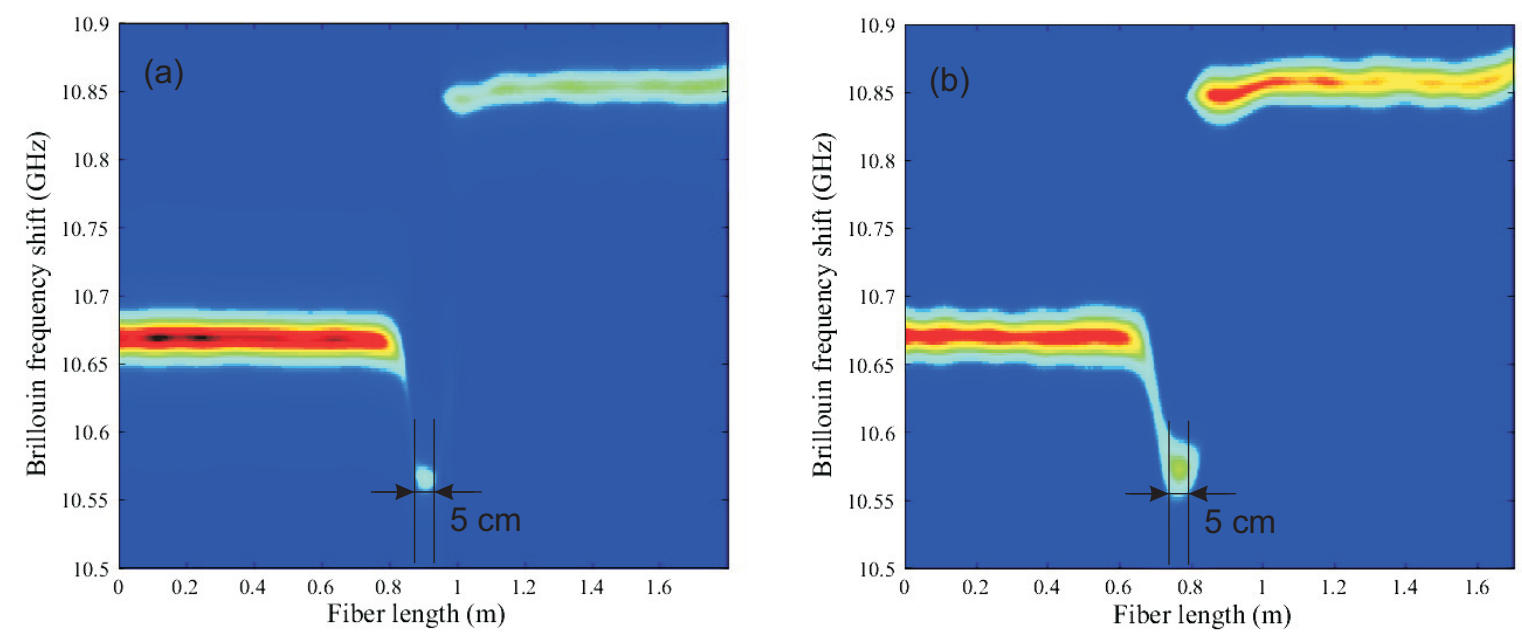

Fig. 3. Distributed measurements of HNA/SMF fibres showing a splice segment of $5 \mathrm{~cm}$ (a) in standard BEDS system using a phase modulator and (b) in our BEDS system based on DPSK technique.

\section{Conclusion}

We have successfully demonstrated a new technique for Brillouin echo-distributed sensing (BEDS) with centimeter resolution based on differential phase-shift keying technique. A $\pi$-phase pulse with a pulse duration of $500 \mathrm{ps}$ and an intensity pulse with $30 \mathrm{~ns}$ are generated using a single Mach-Zehnder modulator. Our results show clearly a spatial resolution of $5 \mathrm{~cm}$ and Brillouin frequency shift at the splice segment between two fibres. Our new technique allows for centimetre resolution by simply adding a negative pulse into Brillouin optical time-domain analysis system. Therefore it simplifies conventional BEDS systems by using a single modulator for pump rather than two modulators, and improves the optical loss of the pump. It also allows an easy adjustment of the delay between two pulses.

\section{Acknowledgements}

The authors acknowledge the European INTERREG IVA programme. They also thank Photline Technologies for providing the Mach-Zehnder modulators used in the work.

\section{References}

1. T. Kurashima, T. Horiguchi, and M. Tateda, "Distributed-temperature sensing using stimulated Brillouin scattering in optical silica fibers," Optics Letters 15(18), pp. 1038-1040, 1990.

2. K. Y. Song, Z. He, and K. Hotate, "Distributed strain measurement with millimeter-order spatial resolution based on Brillouin optical correlation domain analysis," Optics Letters 31(17), pp. 2526-2528, 2006.

3. W. Zou, Z. He, and K. Hotate, "Single-End-Access Correlation-Domain distributed Fiber-Optic sensor based on stimulated Brillouin scattering," Journal of Lightwave Technology 28(18), pp. 2736-2742, 2010.

4. F. Rodríguez-Barrios, S. Martín-López, A. Carrasco-Sanz, P. Corredera, J. D. Ania-Castañón, L. Thévenaz, and M. González-Herráez, "Distributed Brillouin fiber sensor assisted by First-Order Raman amplification," Journal of Lightwave Technology 28(15), pp. 2162-2172, 2010.

5. W. Li, X. Bao, Y. Li, and L. Chen, "Differential pulse-width pair BOTDA for high spatial resolution sensing," Optics Express 16, pp. 21616-21625, 2008.

6. L. Thévenaz, "Brillouin distributed time-domain sensing in optical fibers: state of the art and perspectives," Frontiers of Optoelectronics in China 3, pp. 13-21, 2010.

7. S. M. Foaleng, M. Tur, J. Beugnot, and L. Thévenaz, "High spatial and spectral resolution Long-Range sensing using Brillouin echoes," Journal of Lightwave Technology 28(20), pp. 2993-3003, 2010.

8. G. Charlet, "Progress in optical modulation formats for high-bit rate WDM transmissions," IEEE J. Quantum Electron. 12, pp. 469-483, July 2006. 